\title{
Analysing the Influence of Subsurface Impurity on Laser-induced Damage Threshold of KDP Crystal
}

\author{
Chunyuan Jing ${ }^{\mathrm{a}}$, Xiaohong Guo ${ }^{\mathrm{b}}$, Tao Zhang ${ }^{\mathrm{c}}$, Lizi Chen ${ }^{\mathrm{d}}$ \\ Northwest Institute of Nuclear Technology, Xi'an, Shaanxi, 710024, P.R.China \\ ajingcy59582@sohu.com, ${ }^{b}$ gxh55734@163.com, 'ztao55760@163.com, ${ }^{d}$ Izchen1234@aliyun.com
}

Keywords: KDP, finite element analysis, laser-induced damage threshold, impurity

\begin{abstract}
The laser-induced damage threshold (LIDT) of KDP crystal affect directly the increase of laser power. In order to research the influence of metal impurities in the subsurface of KDP crystal on LIDT, analyzed the impurities composition and relative content of two kinds of KDP crystal samples by fluorescence imaging method. Based on the theory of the thermal conduction equations and the thermal-elastic equations, the LIDT of KDP crystal on finite element method was analyzed. The results indicate that impurities may affect the distribution of temperature field and thermal stress field of the KDP crystal, resulting in a decline in the LIDT of the material, at the same time, different kinds of impurity and the impurity content will also impact on LIDT.
\end{abstract}

\section{Introduction}

KDP crystal is non-linear optical crystal materials with excellent optical properties. The investigation of crystal growth has been lasting for more than 70 years. The type of crystals has been one of the lasting water-soluble crystals and widely applied in the high technology fields such as laser frequency conversion, electro-optic modulation and optical rapid switch etc ${ }^{[2,3]}$. KDP crystal intrinsic absorption of laser is very small ${ }^{[4]}$, but in the process of KDP crystal growth, joined by the organic complexing agent and the coolant used in the manufacturing process of all contains a lot of impurity ions ${ }^{[5,6]}$, thus KDP crystal and the surface in the process of preparing and processing will inevitably contain impurities, these impurities on the absorption of laser is orders of magnitude higher than crystal intrinsic absorption ${ }^{[7]}$, the impurities on the absorption of laser energy is one of the important reasons for KDP crystal damage.

\section{Impurity composition detection}

According to different physical and chemical properties of impurity, the impurities of KDP crystal is usually divided into three categories, respectively is metal cation impurity ${ }^{[8]}$, anion impurity and organic impurity ${ }^{[9,10]}$, the metal cation impurity effects on the optical properties of KDP crystal is the largest among them. In ultraviolet band, because the metal cation has stronger absorption properties, to cause a decline in crystal optical performance. In addition, relative to the pyramidal sector of KDP crystal, the metal cation segregated coefficient of prismatic sector is larger, so in the near ultraviolet band, cylindrical growing area has stronger absorption. For example, $\mathrm{Cr}^{3+}$ easily results in the decrease of crystal in ultraviolet transmittance, high concentrations can lead to intrinsic absorption of the crystal.

This paper analyzed two kinds of KDP crystal samples (traditional method and rapid growth), the samples were taken from the prismatic sector of crystal, were measured by inductively coupled plasma mass spectrometry(ICP-MS). The analysis results are shown in table 1. 
Table1 Amount of metal cation impurities in KDP samples

\begin{tabular}{ccccc}
\hline \multirow{2}{*}{ Impurity } & \multicolumn{2}{c}{ Traditional method } & \multicolumn{2}{c}{ Rapid method } \\
& Sample I & Sample II & Sample I & Sample II \\
\hline $\mathrm{Fe}^{3+}$ & 7.43 & 8.12 & 9.63 & 9.37 \\
$\mathrm{Cr}^{3+}$ & 0.38 & 0.38 & 1.43 & 1.36 \\
$\mathrm{Al}^{3+}$ & 4.33 & 4.21 & 2.76 & 2.75 \\
$\mathrm{Mg}^{2+}$ & 0.07 & 0.05 & 0.04 & 0.04 \\
$\mathrm{Cu}^{2+}$ & 0.05 & 0.05 & 0.17 & 0.16 \\
$\mathrm{~Pb}^{2+}$ & 0.09 & 0.08 & 0.07 & 0.07 \\
$\mathrm{Ba}^{2+}$ & 0.32 & 0.35 & 0.41 & 0.40 \\
$\mathrm{Sr}^{2+}$ & 0.12 & 0.13 & 1.06 & 1.09 \\
\hline
\end{tabular}

According to table 1, we can see that in all samples containing various impurity elements. Through comparison we found that, $\mathrm{Fe}^{3+}, \mathrm{Cr}^{3+}, \mathrm{Al}^{3+}, \mathrm{Ba}^{2+}$, four kinds of impurity ions content are more other elements. In the latter part of this article, the eight kinds of impurity element distribution of temperature field and thermal stress field distribution will be analyzed.

\section{Simulation Modeling Method}

Due to the absorption of KDP crystal is very small, when KDP crystal surface by laser irradiation, the temperature change is not big, but due to the impurities of incident laser energy of strong absorption, formation temperature gradient will produce thermal stress in the crystal. If the temperature reaches melting point, impurity will cause the additional stress distribution change inside the crystal. When crystals in stress than material tensile or compressive strength, can make the material cracking or crushed.

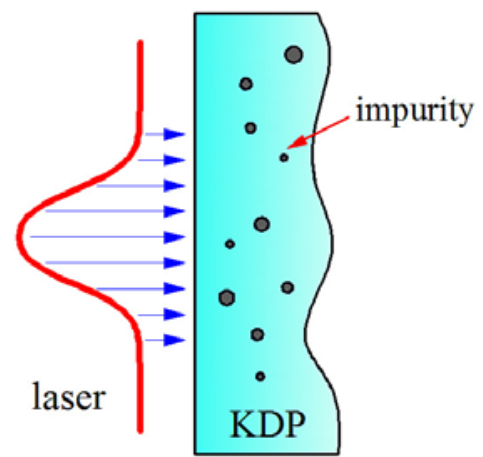

Fig.1 KDP crystal with impurities irradiated by laser

Assumes that the impurity is spherical in KDP crystal, suppose laser irradiation on the crystal surface, the light intensity distribution is gaussian distribution. Due to the radius of the impurities are usually about nano or micro level, and the crystal size is at least millimeter level. Therefore, impurities can be seen as in the infinite crystal materials, mainly considering the absorption of impurities and ignore the absorption of crystal in simulation.

$$
\left\{\begin{array}{lc}
\rho_{i} \frac{\partial\left(c_{i} T\right)}{\partial t}=\frac{1}{r^{2}} \frac{\partial}{\partial r}\left(r^{2} k_{i} \frac{\partial T}{\partial r}\right)+q, & r<r_{0}, t \geq 0 \\
T_{i}=T_{s}, k_{i} \frac{\partial T_{i}}{\partial r}=k_{s} \frac{\partial T_{s}}{\partial r}, & r=r_{0} \\
\rho_{s} \frac{\partial\left(c_{s} T\right)}{\partial t}=\frac{1}{r^{2}} \frac{\partial}{\partial r}\left(r^{2} k_{s} \frac{\partial T}{\partial r}\right), & r>r_{0}, t \geq 0
\end{array}\right.
$$

where $\rho$ is the material density, $c$ is the specific heat, $k$ is the heat conduction, $q$ is the internal heat source, $r_{0}$ is the radius of the impurity, the subscript $i$ and $s$ represent the impurities and KDP crystal. The $q$ can be expressed as follows form ${ }^{[11]}$ : 


$$
q=3 \eta I / 4 r_{0}
$$

where $\eta$ is impurity absorption coefficient, $I$ is laser fluence.

As a spherically symmetric thermal stress problem, both of $\theta$ and $\phi$ are the same in the thermal elastic constitutive equation. The thermal stress in spherical coordinates of the equation can be expressed as:

$$
\left\{\begin{array}{l}
u_{r}=\frac{1+v}{1-v} \frac{\alpha}{r^{2}} \int_{r}^{r_{0}} \operatorname{Tr}^{2} d r+c_{1} r+\frac{c_{2}}{r^{2}} \\
\sigma_{r r}=-\frac{2 \alpha E}{1-v} \frac{1}{r^{3}} \int_{r}^{r_{0}} \operatorname{Tr}^{2} d r+\frac{E c_{1}}{1-2 v}-\frac{2 E c_{2}}{1+v} \frac{1}{r^{3}} \\
\sigma_{r r}=\frac{\alpha E}{1-v} \frac{1}{r^{3}} \int_{r}^{r_{0}} \operatorname{Tr}^{2} d r+\frac{E c_{1}}{1-2 v}+\frac{E c_{2}}{1+v} \frac{1}{r^{3}}-\frac{\alpha E T}{1-v}
\end{array}\right.
$$

where $E$ is elasticity modulus of crystal, $v$ is Poisson's ratio of crystal, $\alpha$ is coefficient of thermal expansion.

\section{Results and Discussion}

This paper established finite element model of KDP crystal with a single impurity ions, mainly analyzes the distribution of temperature field and thermal stress field near the impurities. when coaxial gaussian laser beam incident on the crystal surface, considering the calculation process is convenient, the finite element model only selected the $1 / 2$ of the crystals as computing subject. It is hypothesized that KDP crystal diameter is $5 \mathrm{~mm}$, thickness of $10 \mathrm{~mm}$, the surface reflection coefficient is 0.05 , and except the top surface of glass (the heat flux on the top surface simulates convective cooling), all other boundaries are assumed to be thermally insulated, impurity is spherical, radius of $0.1 \mu \mathrm{m}$, the distance between the laser incident surface is $100 \mu \mathrm{m}$.

Images presented in Fig. 2 show that the surface temperature changes of iron ion impurities under the $5 \mathrm{~ms}$ single pulse laser irradiation, laser beam focus spot radius is $0.3 \mathrm{~mm}$, the average laser fluence of single pulse is $20 \mathrm{~J} / \mathrm{cm}^{2}$, wavelength is $1064 \mathrm{~nm}$.

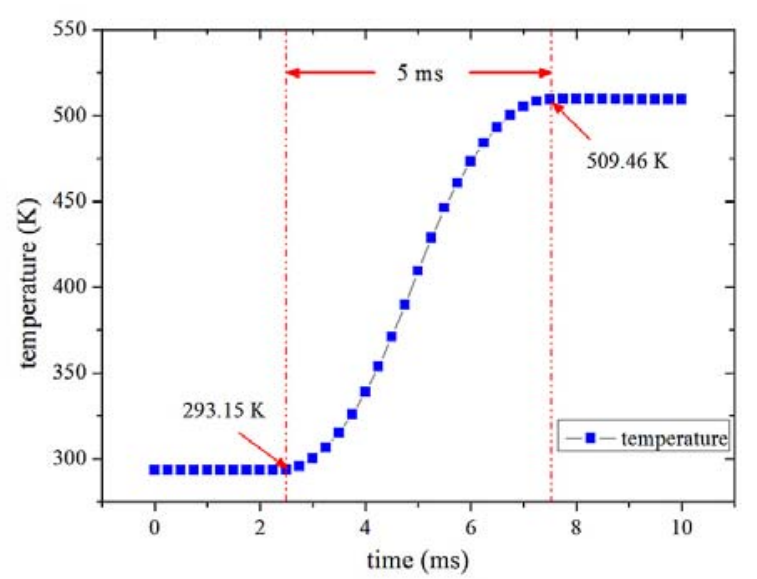

Fig. 2 Impurity surface temperature changes over time $\left(\mathrm{Fe}^{3+}\right)$

In the Fig.2, the red areas for single pulse laser irradiation time( $5 \mathrm{~ns})$, the initial temperature of crystal and impurity is $293.15 \mathrm{~K}$, at the end of the laser pulse energy, the temperature of impurity surface reached maximum, is $509.46 \mathrm{~K}$.

Image presented in Fig. 3 show that the temperature and thermal stress field distribution curve around the $\mathrm{Fe}^{3+}$ ion. In the Fig.3(a) we can see that the temperature is basically the same within the radius of impurity, the impurity near the crystal temperature change is very small, and away from the impurity, the temperature of crystal drop very quickly. 

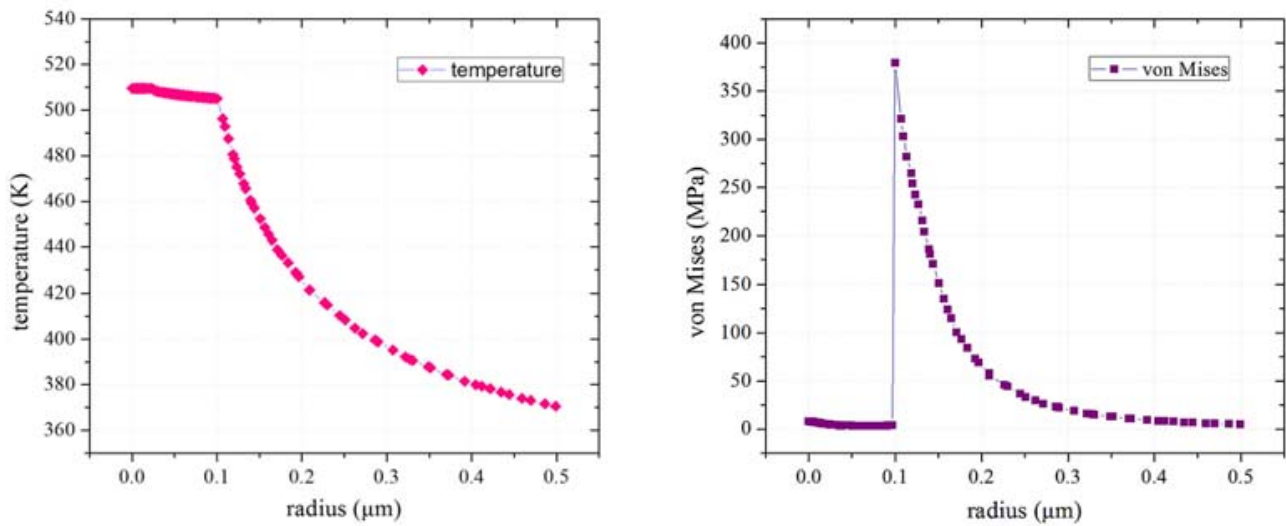

Fig. 3 Temperature and thermal stress field distribution curve around the impurity $(\mathrm{Fe} 3+)$ of KDP crystals: (a) temperature field distribution curve; (b) thermal stress field distribution curve.

Images presented in Fig.3(b) show that thermal stress field distribution curve around the $\mathrm{Fe}^{3+}$ ion. Due to impurity $\mathrm{Fe}^{3+}$ on laser energy of strong absorption, dramatic expansion in the impurity result in on the border of impurities and crystal generate strong stress, impurity near the maximum thermal stress for $\sigma=379.2 \mathrm{MPa}$, the value is beyond the yield strength of KDP crystal (360MPa), cause the material to produce thermal stress damage. These phenomena indicate that impurities of crystal temperature only focused on the influence of impurity near a small area, which due to the impurity of laser with strong absorption, the temperature of the impurity sharp increase lead to strong thermal stress, it can make the crystal material damage.
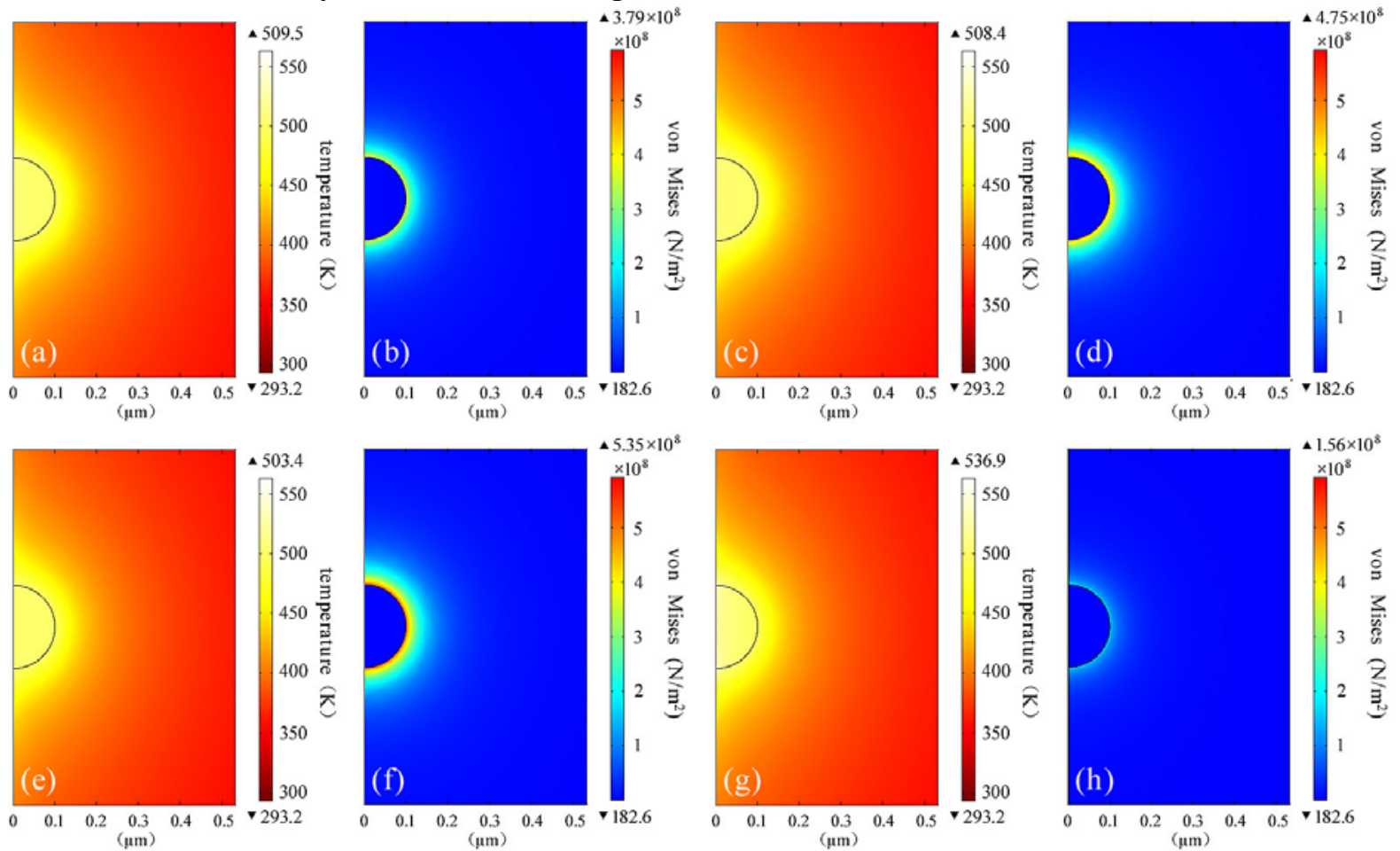

Fig.4 Temperature field and thermal stress distribution around the impurity:(a),(b) $\mathrm{Fe}^{3+} ;$ (c),(d) $\mathrm{Cr}^{3+} ;$ (e),(f) $\mathrm{Al}^{3+}$; (g), (h) $\mathrm{Ba}^{2+}$.

In addition to the temperature and thermal stress field distribution of $\mathrm{Fe}^{3+}$ ion were analyzed, also analyzed other content relatively high metal ions, is shown in Fig.4. We assume that the average laser fluence of single pulse $(5 \mathrm{~ms})$ is $20 \mathrm{~J} / \mathrm{cm}^{2}$. From the Fig.4, can be found that $\mathrm{Fe}^{3+}, \mathrm{Cr}^{3+}, \mathrm{Al}^{3+}$, $\mathrm{Ba}^{2+}$, four kinds of ion of the highest temperature is only about $500 \mathrm{~K}$, far from achieving their melting point. But in addition to $\mathrm{Ba}^{2+}$, the maximum thermal stress of other three kinds of ion is more than the yield stress of KDP crystal. The thermal stress of $\mathrm{Al}^{3+}$ ion is the largest among them, reached $535 \mathrm{MPa}$. Although the content of $\mathrm{Fe}^{3+}$ ion is the most, it generated by the thermal stress is much smaller than $\mathrm{Al}^{3+}$. 

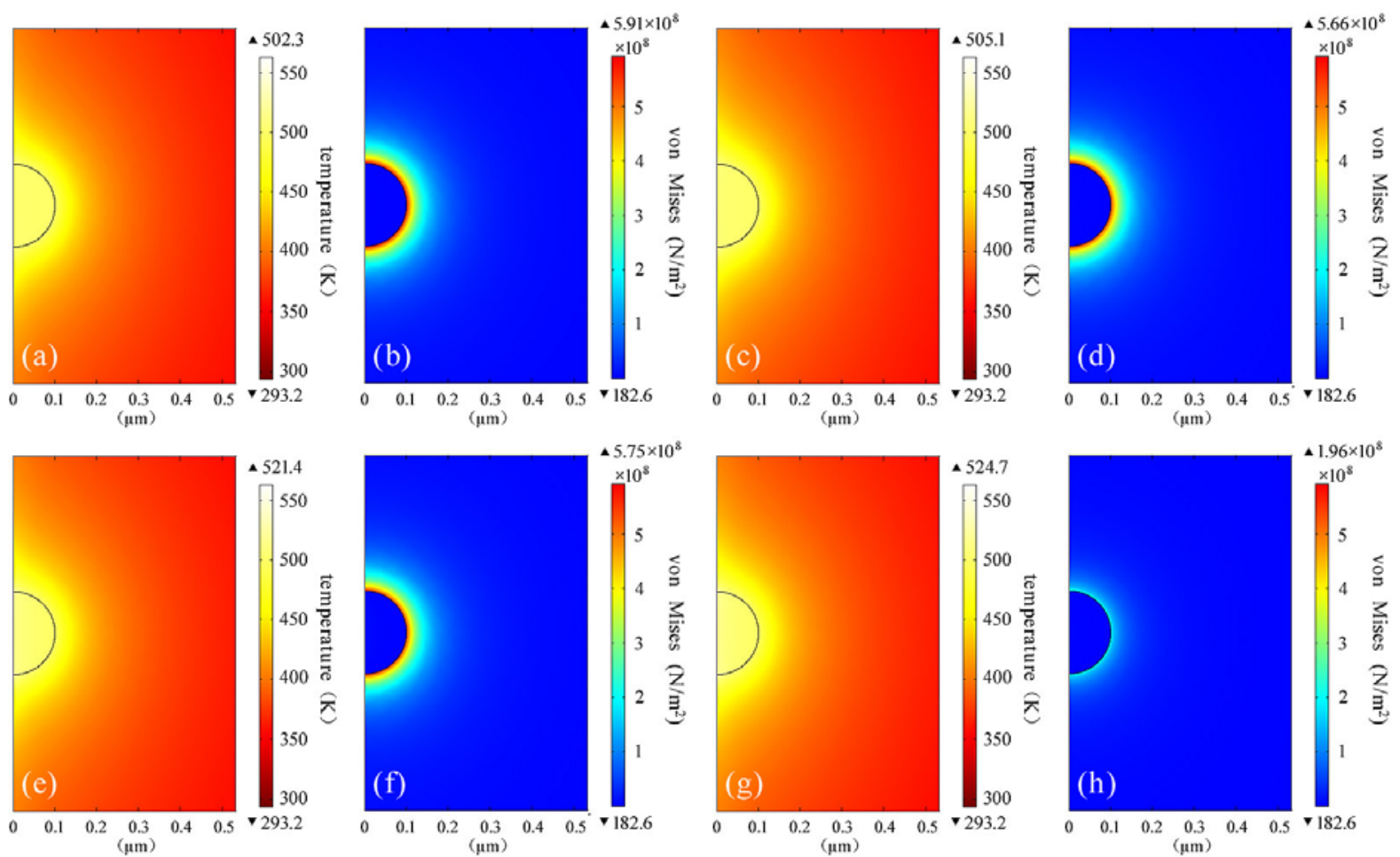

Fig.5 Temperature field and thermal stress distribution around the impurity:(a),(b) $\mathrm{Cu}^{2+} ;(\mathrm{c}),(\mathrm{d}) \mathrm{Pb}^{2+} ;(\mathrm{e}),(\mathrm{f}) \mathrm{Mg}^{2+}$; (g), (h) $\mathrm{Sr}^{2+}$.

Using the same method, we analyzed the temperature field and thermal stress distribution of other four kinds of metal ions. $\mathrm{Cu}^{2+}, \mathrm{Pb}^{2+}, \mathrm{Mg}^{2+}$, the three ions generated by thermal stress than crystal yield limit, and more than aluminum ions produced by thermal stress but the content of these ions are relatively low, so cause material laser induced damage probability is also smaller.

\section{Conclusion}

This paper measured the metal ion content of KDP crystal by inductively coupled plasma mass spectrometry, and established the finite element model of KDP crystal, mainly analyzes the distribution of temperature field and thermal stress field near the impurities. The results indicate that impurity absorption of laser energy is much higher than the intrinsic absorption of KDP crystal, the temperature rise of the impurity is very severe, produce very large thermal stress, impact on KDP crystals laser damage threshold. In addition, in several content relatively high metal ions, the aluminum ions had the greatest influence on KDP crystals laser damage threshold. At the same time, $\mathrm{Cr}^{3+}, \mathrm{Cu}^{2+}, \mathrm{Pb}^{2+}, \mathrm{Mg}^{2+}$ also has great influence, therefore these ions in the crystal process should decrease as far as possible.

\section{References}

[1] Fujioka K, Matsuo S, Kanabe T, et al. Optical properties of rapidly grown KDP crystal improved by thermal conditioning[J]. Journal of crystal growth, 1997, 181(3): 265-271.

[2] Rajesh P, Ramasamy P, Bhagavannarayana G. Growth of ADP-KDP mixed crystal and its optical, mechanical, dielectric, piezoelectric and laser damage threshold studies[J]. Journal of Crystal Growth, 2013, 362: 338-342.

[3] De Yoreo J J, Burnham A K, Whitman P K. Developing KH2PO4 and KD2PO4 crystals for the world's most power laser[J]. International Materials Reviews, 2002, 47(3): 113-152.

[4] Hopper R W, Uhlmann D R. Mechanism of inclusion damage in laser glass[J]. Journal of Applied Physics, 2003, 41(10): 4023-4037. 
[5] Miki H, Terashima T, Asakuma $\mathrm{Y}$, et al. Inclusion of mother liquor inside KDP crystals in a continuous MSMPR crystallizer[J]. Separation and purification technology, 2005, 43(1): 71-76.

[6] Sun X, Xu X G, Sun D L, et al. Study on the liquid inclusion induced light scatter in KDP crystal[J]. Journal of crystal growth, 2001, 226(4): 529-533.

[7] Nishida Y, Yokotani A, Sasaki T, et al. Improvement of the bulk laser damage threshold of potassium dihydrogen phosphate crystals by reducing the organic impurities in growth solution[J]. Applied physics letters, 1988, 52(6): 420-421.

[8] Land T A, Martin T L, Potapenko S, et al. Recovery of surfaces from impurity poisoning during crystal growth[J]. Nature, 1999, 399(6735): 442-445.

[9] Li G, Liping X, Su G, et al. Study on the growth and characterization of KDP-type crystals[J]. Journal of crystal growth, 2005, 274(3): 555-562.

[10]X. M. Mu, S. L. Wang, X. G. Xu, et al. Optical Properties and Structural Perfection of Chicago Sky Blue 6B-doped KDP Crystals[J]. Chinese J. Struct. Chem, 2010, 29: 121-125.

[11]Hopper R W, Uhlmann D R. Mechanism of inclusion damage in laser glass[J]. Appl Phys, 1970, 41(10): 4023-4037. 